\title{
UTERINE NATURAL KILLER CELLS IN PROLIFERATIVE ENDOMETRIUM OF INFERTILE WOMEN
}

\author{
Prof G. Menaldo, Prof S. Serrano, Dr A. Giovannelli, Dr B. Lopez \\ Centro Clinico San Carlo di Torino, Unità di Procreazione Assistita, Torino, Italy
}

INTRODUCTION: Embryo implantation is influenced by local and systemic immune responses involving immunoglobulins, cytokines, hormonal and other endometrial factors. A synergism of these factors is critical for successful implantation and subsequent conception. Natural killer (NK) cells have been implicated to play a role in female reproductive performance. They have been thought to be associated with implantation failures, recurrent miscarriage or infertility due to either NK cell cytotoxicity or receptor/gene expression.

NK cells cause cytotoxic effects by inducing lysis or apoptosis of the target cells mediated by the release of granular components within their cytoplasm or secretion of cytokines, such as tumor necrosis factor-alpha, interleukin 10 , interferon -gamma and transforming growth factor -beta.

The human endometrium contains a substantial population of NK cells which vary in number and in proportion to the total number of endometrial stromal cells during the menstrual cycle. NK cells increase in number substantially in the mid-secretory phase and are the major endometrial lymphocyte population in the late secretory phase and the first trimester of pregnancy. Uterine NK cells are CD56, CD16 and also CD138+ for the diagnosis of endometritis.

The regulation of uterine natural killer (NK) cells has been associated with reproductive conditions including recurrent pregnancy loss (RPL) and implantation failure.

It is speculated that in women an elevation of natural killer (NK) cells may have an effect on reproductive performance.

Women who have fertility problems, specifically miscarriage or unsuccessful IVF are more likely to have higher levels of activity of these 'Natural Killer cells' than other women.

The aim of our study was to analyze NK cell concentration in the proliferative endometrium (before ovulation around day 10) of infertile patients and NK cell levels in endometrium are currently being used as a diagnostic test to guide the initiation of therapies in patients with infertility.

METHODS: This study was conducted with 63 patients with unexplained infertility. Proliferative endometrial tissue samples were obtained with a Pipelle catheter, Performing local endometrial injury (endometrial scratching) and the endometrial CD 16, CD 56 and CD138+ were determined.

Endometrial scratching seems to provoke a reaction within the inner lining of the womb. Hormones and chemicals are released to help the lining repair itself. A genetic trigger response to an endometrial scratch may give the implantation 'green light'. In essence, the temporary injury seems to make the endometrium more receptive to an embryo. That means a better chance of a pregnancy and a live birth.

RESULTS: Immunologic analyses are increasingly implemented in infertility diagnostics. One of the parameters of interest are uterine NK cells. Our study reveals presence of CD16 CD56 and CD138 in the proliferative endometrial tissue of infertile women. It were considered positive cases presence of a number of cells of $C D>10$ cells.

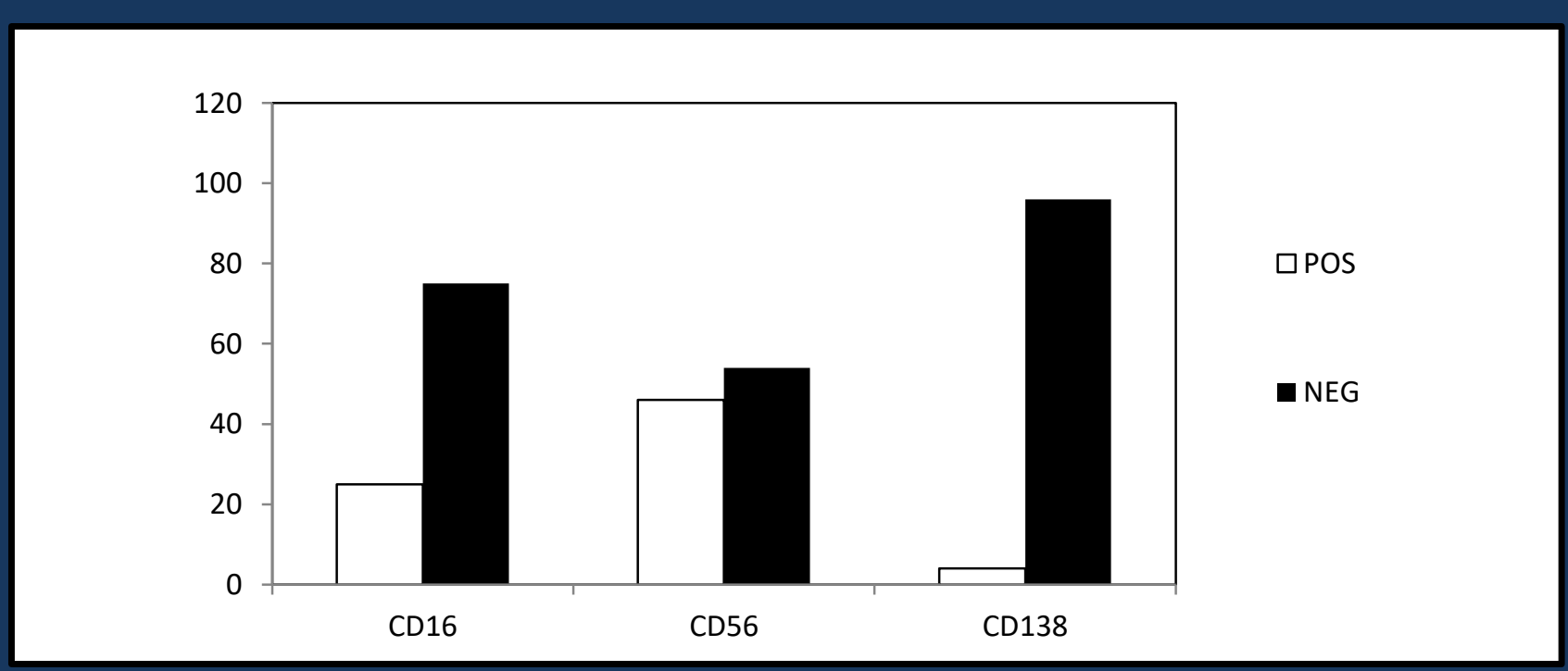

CD16 and CD56 cell abundance in the proliferative endometrial tissue of women with reproductive failure has suggested they may play a role in this pathogenesis. Common treatment for women with abnormal endometrial NK cells numbers is use of corticosteroids but it has not shown a really efficacy. For this reason in our lab we use a biophotonic therapy: as described in our previous study, through a special device connected to the computer, the woman received the spermatic biophotonic emission of her male partner, previously stored. The administration of spermatic biophotonic energy was carried out a few minutes before the intratubal insemination in acupuncture points and at pelvic and vaginal level, and on culture medium in which there are incubated embryos obtained through ICSI et/or PICSI. The pregnancy rate was significantly higher in this group of patients.

Furthermore, performing this combined treatment with endometrial scratching in patients with a diagnosis of infertility increased the pregnancy and clinical pregnancy rates. 